\title{
E(5) and X(5) shape phase transitions within a Skyrme-Hartree-Fock + BCS approach
}

\author{
R. Rodríguez-Guzmán and P. Sarriguren* \\ Instituto de Estructura de la Materia, CSIC, Serrano 123, E-28006 Madrid, Spain
}

(Received 11 October 2007; published 5 December 2007)

\begin{abstract}
Self-consistent Skyrme-Hartree-Fock plus BCS calculations are performed to generate potential energy curves (PECs) in various chains of $\mathrm{Pd}, \mathrm{Xe}, \mathrm{Ba}, \mathrm{Nd}, \mathrm{Sm}, \mathrm{Gd}$, and Dy isotopes. The evolution of shapes with the number of nucleons is studied in a search for signatures of $\mathrm{E}(5)$ and $\mathrm{X}(5)$ critical point symmetries. It is shown that the energy barriers in the PECs are determined to a large extent by the treatment of the pairing correlations.
\end{abstract}

DOI: 10.1103/PhysRevC.76.064303

PACS number(s): 21.60.Jz, 21.60.Fw, 27.60.+j, 27.70.+q

\section{INTRODUCTION}

The ground states of atomic nuclei are characterized by different equilibrium configurations which correspond to different geometrical shapes. The study of these equilibrium shapes, as well as the transition regions between them, has been the subject of a large number of theoretical and experimental studies (for a review, see, for example, Ref. [1] and references therein).

Within the framework of algebraic models, the different nuclear phase shapes are put in correspondence to dynamic symmetries of some algebraic structure that links a specific mathematical symmetry with a specific nuclear shape. Dynamic symmetries provide a useful tool for describing properties of different physical systems, since they lead to exactly solvable problems and produce all results for observables in explicit analytic form.

In nuclear physics, the algebraic structure of relevance, according to the interacting boson model (IBM) [2], is given by $\mathrm{U}(6)$. There are three dynamic symmetries characterized by $\mathrm{U}(5)$, which is associated with spherical symmetry; $\mathrm{SU}(3)$, which is associated with axially deformed symmetry; and $\mathrm{SO}(6)$, which is associated with describing $\gamma$-unstable shapes. Experimental examples of all three types of symmetries have been recognized in many nuclei.

The phase shape transitions correspond to the breaking of these dynamic symmetries, and they occur as the number of nucleons change in the nucleus. Understanding the behavior of systems undergoing a phase transition is of special relevance, since a complicated interplay of competing degrees of freedom occurs at the critical points.

Iachello $[3,4]$ introduced the $E(5)$ and $X(5)$ critical point symmetries within the framework of the collective Bohr Hamiltonian [5] under some simplifying approximations. Critical point symmetries provide parameter-free (up to scale factors) predictions of excitation spectra and electric quadrupole $B(E 2)$ strengths for nuclei at the critical point of a phase shape transition. The geometrical shape of the ground state can be described [5] by three Euler angles and by the quadrupole deformation parameters $\beta$ and $\gamma$. At the critical point of the phase transition, the potential in the $\beta$

*sarriguren@iem.cfmac.csic.es degree of freedom can be approximated by a simple square well potential, which is decoupled from the potential in the $\gamma$ variable. In the case of the $\mathrm{E}(5)$ critical point symmetry, which corresponds to the transition from spherical vibrational $\mathrm{U}(5)$ to deformed $\gamma$-unstable $\mathrm{O}(6)$, the potential is flat in the $\gamma$ direction. In the case of the symmetry $\mathrm{X}(5)$, related to the transition from $\mathrm{U}(5)$ to axially symmetric prolate $\mathrm{SU}(3)$, a harmonic oscillator potential is used in the $\gamma$ direction.

Empirical evidence of these transitional symmetries at the critical points were soon found in ${ }^{134} \mathrm{Ba}$ [6] for $\mathrm{E}(5)$ and in ${ }^{152} \mathrm{Sm}$ [7] for $\mathrm{X}(5)$. Other nuclei have been also identified as good candidates for those symmetries. Such is the case of ${ }^{102} \mathrm{Pd}$ [8] and ${ }^{128-130} \mathrm{Xe}$ [9], which provide examples of $\mathrm{E}(5)$ symmetry. Other $N=90$ isotones such as ${ }^{150} \mathrm{Nd}$ [10], ${ }^{154} \mathrm{Gd}[11]$, and ${ }^{156} \mathrm{Dy}$ [12] also provide examples of $\mathrm{X}(5)$ symmetry.

Algebraic models are very suitable for systematic studies, because they provide powerful predictions with a very small number of parameters; but to deepen into the details, one has to perform microscopic investigations of shape transitions and critical points which are, to a large extent, still missing. In particular, it is interesting to examine whether the assumptions of relatively flat potentials in $\mathrm{E}(5)$ and $\mathrm{X}(5)$ are justified in different microscopic models; the self-consistent mean-field approximation, based on parametrizations widely used all over the nuclear chart, appears as a very attractive initial tool for linking algebraic models and microscopic theories. In this context, the relativistic mean-field framework has been employed in calculations of potential energy curves (PECs) as functions of the quadrupole deformation [13-15]. These studies have been performed for isotopic chains in which the occurrence of critical point symmetries has been predicted. Since flat PECs are one of the expected characteristics of critical point symmetries, constrained calculations in those isotopic chains should result in relatively flat PECs for nuclei with the critical symmetry. It has been shown that particular isotopes exhibit relatively flat PECs over an extended range of the deformation parameter. Nevertheless, the behavior of the PECs, and particularly the potential barriers, are quite sensitive to the relativistic interaction used [13], as well as to the pairing treatment. Therefore, the question arises whether a similar situation occurs when using nonrelativistic effective interactions to study candidates for critical point symmetries. Such systematic nonrelativistic studies are still missing, and it is very interesting to compare the conclusions extracted from 
them with those obtained using both algebraic models and relativistic mean-field approximations.

In this work, we extend the calculations mentioned above to the case of nonrelativistic self-consistent SkyrmeHartree-Fock + BCS mean-field calculations. The shape phase transitions corresponding to $\mathrm{E}(5)$ and $\mathrm{X}(5)$ symmetries are investigated systematically in various isotopic chains containing some of the suggested critical nuclei. In particular, we study $\mathrm{Pd}, \mathrm{Xe}$, and $\mathrm{Ba}$ isotopic chains as candidates of E(5) symmetry, and $\mathrm{Nd}, \mathrm{Sm}, \mathrm{Gd}$, and Dy isotopic chains as examples of $\mathrm{X}(5)$.

The paper is organized as follows. In Sec. II, we present a brief description of the theoretical formalism (HartreeFock+BCS) used to obtain the main ingredient of the present study, i.e., the PECs for the considered isotopic chains. For a more detailed account the reader is referred to the corresponding literature. Section III contains our results with a discussion on the sensitivity of the PECs to the effective nucleon-nucleon force and to the treatment of the pairing correlations. Section IV is devoted to the concluding remarks.

\section{THEORETICAL FRAMEWORK}

The microscopic approach used in this work (i.e., $\mathrm{HF}+\mathrm{BCS}$ ) is based on a self-consistent formalism built on a deformed Hartree-Fock (HF) mean-field, using Skyrme-type energy density functionals. Pairing correlations between like nucleons are included in the BCS approximation. It is well known that the density-dependent $\mathrm{HF}+\mathrm{BCS}$ approximation provides a very good description of ground-state properties for both spherical and deformed nuclei, [16] and it is at present one of the state-of-the-art mean-field descriptions [17].

There are two leading choices of methods to solve the deformed $\mathrm{HF}+\mathrm{BCS}$ equations. One option is the use of a coordinate space mesh. In this case, one solves the HF+BCS equations for Skyrme-type functionals via discretization of the individual wave functions on a three-dimensional Cartesian mesh [18]. This corresponds to an expansion on a specific basis of Lagrange polynomials associated with the selected mesh. The other common choice is to expand the single-particle wave functions into an appropriate orthogonal basis (commonly the eigenfunctions of an axially symmetric harmonic oscillator potential). In the present study, we perform calculations with both methods. We use the code EV8 [18] in the first case and follow the procedure based on the formalism developed in Ref. [19] in the second case.

In this work, we consider the parametrization SLy4 [20] of the Skyrme force in the particle-hole channel, although we also show results in some instances for the forces Sk3 [21] and SG2 [22]. They are examples of global effective interactions of Skyrme-type that have been designed to fit ground state properties of spherical nuclei and nuclear matter properties. While Sk3 is the most simple one, involving in particular a linear dependence on the density, SLy4 is one of the most recent parametrizations of Skyrme forces.

As we move away from closed shells, pairing correlations play an important role [23] and should be taken into account. If one were dealing with a fundamental many-body Hamiltonian, one would proceed to apply Hartree-Fock-Bogoliubov formalism to it. However, dealing with Skyrme forces that have been simplified with the aim of reproducing average or bulk properties of the nucleus, one would have to include additional parameters to guarantee that sensible pairing matrix elements are obtained $[17,18]$.

In this study, pairing correlations are taken into account in the BCS approximation. Several options have been investigated. Our main option is the use of a zero-range densitydependent pairing force [24],

$$
V\left(\mathbf{r}_{1}, \mathbf{r}_{2}\right)=-g\left(1-\hat{P}^{\sigma}\right)\left(1-\frac{\rho\left(\mathbf{r}_{1}\right)}{\rho_{c}}\right) \delta\left(\mathbf{r}_{1}-\mathbf{r}_{2}\right),
$$

where $\hat{P}^{\sigma}$ is the spin exchange operator, $\rho(\mathbf{r})$ is the nuclear density, and $\rho_{c}=0.16 \mathrm{fm}^{-3}$. The strength $g$ of the pairing force [Eq. (1)] is taken as $g=1000 \mathrm{MeV} \mathrm{fm}^{3}$ for both neutrons and protons, and a smooth cutoff of $5 \mathrm{MeV}$ around the Fermi level has also been used. Let us mention that very recently the parametrization SLy4 has been successfully applied in combination with the pairing interaction [Eq. (1)] (with $g=1000 \mathrm{MeV} \mathrm{fm}^{3}$ ) in systematic studies of correlation energies from ${ }^{16} \mathrm{O}$ to the superheavies [25] and in global studies of spectroscopic properties of the first $2^{+}$states in even-even nuclei [26]. This is the main reason for selecting the combination SLy4 in the particle-hole channel and the interaction [Eq. (1)] (with $g=1000 \mathrm{MeV} \mathrm{fm}^{3}$ ) in the pairing channel as the leading choice for the present study. Additionally, results with the strength $g=1250 \mathrm{MeV} \mathrm{fm}^{3}$ will also be shown in some cases.

Another common practice for including pairing correlations is to introduce a schematic seniority pairing force with a constant pairing strength $G$ [23], we call this treatment the constant-force approach,

$$
V_{\text {pair }}=-G \sum_{m, m^{\prime}>0} a_{m}^{\dagger} a_{\bar{m}}^{\dagger} a_{\bar{m}^{\prime}} a_{m^{\prime}} .
$$

The strength of the pairing force for protons and neutrons $G_{p, n}$ is chosen in such a way that the experimental pairing gaps extracted from binding energies in neighboring nuclei are reproduced. One can further simplify the pairing treatment by parametrizing the pairing gaps $\Delta_{p, n}$ directly from experiment, we call this treatment the constant-gap approach. The pairing strength and pairing gap are related through the gap equation [23]

$$
\Delta=G \sum_{\nu>0} u_{\nu} v_{\nu}
$$

where $v_{v}$ are the occupation amplitudes.

The PECs shown in this study are computed microscopically by constrained $\mathrm{HF}+\mathrm{BCS}$ calculations $[18,23,27]$. These PECs are obtained by minimizing the corresponding energy functional under a quadratic constraint that holds the nuclear quadrupole moment fixed to a given value. In this work, we show the energies as a function of the (axially symmetric) mass quadrupole moment $Q$, which is related with the quadrupole deformation parameter $\beta_{2}$ by the expression

$$
\beta_{2}=\sqrt{\frac{\pi}{5}} \frac{Q}{A\left\langle r^{2}\right\rangle}
$$


TABLE I. Quadrupole deformation parameters $\beta_{2}$ for the ground states in Pd, Xe, and Ba isotopes. SLy4 force and two values of the pairing strength $g\left(\mathrm{MeV} \mathrm{fm}^{3}\right)$ are used.

\begin{tabular}{lcccccccrrr}
\hline \hline $\mathrm{Pd}$ & 96 & 98 & 100 & 102 & 104 & 106 & 108 & 110 & 112 & 114 \\
$g=1000$ & 0.10 & 0.12 & 0.15 & 0.17 & 0.18 & 0.17 & 0.17 & 0.18 & 0.19 & -0.19 \\
$g=1250$ & 0.00 & 0.10 & 0.14 & 0.15 & 0.16 & 0.16 & 0.15 & 0.13 & 0.13 & 0.11 \\
& & & & & & & & & \\
$\mathrm{Xe}$ & 118 & 120 & 122 & 124 & 126 & 128 & 130 & 132 & 134 & 136 \\
$g=1000$ & 0.15 & 0.29 & 0.29 & 0.23 & 0.20 & 0.17 & 0.14 & 0.11 & 0.08 & 0.00 \\
$g=1250$ & 0.09 & 0.27 & 0.27 & 0.20 & 0.18 & 0.15 & 0.13 & 0.09 & -0.01 & 0.00 \\
$\mathrm{Ba}$ & 120 & 122 & 124 & 126 & 128 & 130 & 132 & 134 & 136 & 138 \\
$g=1000$ & 0.34 & 0.28 & 0.27 & 0.25 & 0.23 & 0.20 & 0.17 & 0.13 & 0.11 & 0.00 \\
$g=1250$ & 0.31 & 0.29 & 0.27 & 0.24 & 0.22 & 0.19 & 0.15 & 0.12 & 0.08 & 0.00 \\
\hline \hline
\end{tabular}

in terms of the mean square radius of the mass distribution $\left\langle r^{2}\right\rangle$. Tables I and II contain the quadrupole parameters $\beta_{2}$ corresponding to the equilibrium configurations for the isotopes considered in this work.

\section{DISCUSSION OF RESULTS}

It is known that PECs are sensitive to the effective nuclear force in both relativistic [13] and nonrelativistic [28,29] approaches, as well as to pairing correlations [28,29]. Thus, it is worth starting our discussion on PECs by studying this sensitivity in our case. We perform this study on the examples of ${ }^{128} \mathrm{Xe}$ and ${ }^{152} \mathrm{Sm}$, which are proposed candidates for $\mathrm{E}(5)$ and $\mathrm{X}(5)$ symmetries, respectively.

In Fig. 1, we study the effect of using different methods of solving the $\mathrm{HF}+\mathrm{BCS}$ equations, coordinate lattice [18] and deformed harmonic oscillator basis [19]. In these calculations, we use the same SLy4 force in the particle-hole channel and the same constant-gap treatment of pairing. The results indicate that the PECs obtained are practically the same in these two cases and, in general, in all the isotopes we have considered in this work.

In Fig. 2, we consider the PECs obtained from three different Skyrme forces, namely, SLy4, SG2, and Sk3, using the same pairing treatment. We can see that the location of the oblate and prolate minima appear at the same deformation

TABLE II. Quadrupole deformation parameters $\beta_{2}$ for the ground states in $\mathrm{Nd}, \mathrm{Sm}, \mathrm{Gd}$, and Dy isotopes. SLy4 force and pairing strength $g=1000 \mathrm{MeV} \mathrm{fm}^{3}$ are used.

\begin{tabular}{lcccccccc}
\hline \hline Nd & 142 & 144 & 146 & 148 & 150 & 152 & 154 & 156 \\
& 0.00 & 0.10 & 0.14 & 0.20 & 0.28 & 0.29 & 0.29 & 0.30 \\
Sm & 144 & 146 & 148 & 150 & 152 & 154 & 156 & 158 \\
& 0.00 & 0.07 & 0.16 & 0.20 & 0.25 & 0.29 & 0.30 & 0.31 \\
& 146 & 148 & 150 & 152 & 154 & 156 & & \\
Gd & 140 & -0.05 & 0.17 & 0.22 & 0.25 & 0.28 & & \\
& 0.00 & & & & & & & \\
Dy & 148 & 150 & 152 & 154 & 156 & 158 & & \\
& 0.00 & -0.08 & -0.14 & 0.21 & 0.26 & 0.28 & & \\
\hline \hline
\end{tabular}

no matter what the force is. However, the relative energies of the minima and the energy barriers between the minima can change by a few $\mathrm{MeV}$, depending on the force.

In Fig. 3, we show the effect of pairing using SLy4 force in all cases. We consider the constant-gap and constant-force approaches. Again, we observe that the minima appear at about the same deformations, but the energy barriers can change considerably depending on the approach used.

From the analysis of Figs. 1-3, we conclude that, at least in the mass region studied, the PECs are not sensitive to the method employed to solve the $\mathrm{HF}+\mathrm{BCS}$ equations (three-dimensional Cartesian lattice or deformed harmonic oscillator). We also conclude that the qualitative behavior of the energy profiles remains unchanged against changes in the Skyrme and pairing interactions in the sense that the deformations at which the minima occur are rather stable. Nevertheless, the relative energies of these minima and particularly the energy barriers between them are very sensitive to the details of the calculation, especially to pairing. This is also the case in relativistic mean-field calculations. The sensitivity of the PECs to the force can be seen in Ref. [13], where several forces were compared (NL1, NL3, NLSH, and TM1). The sensitivity to the pairing in relativistic calculations
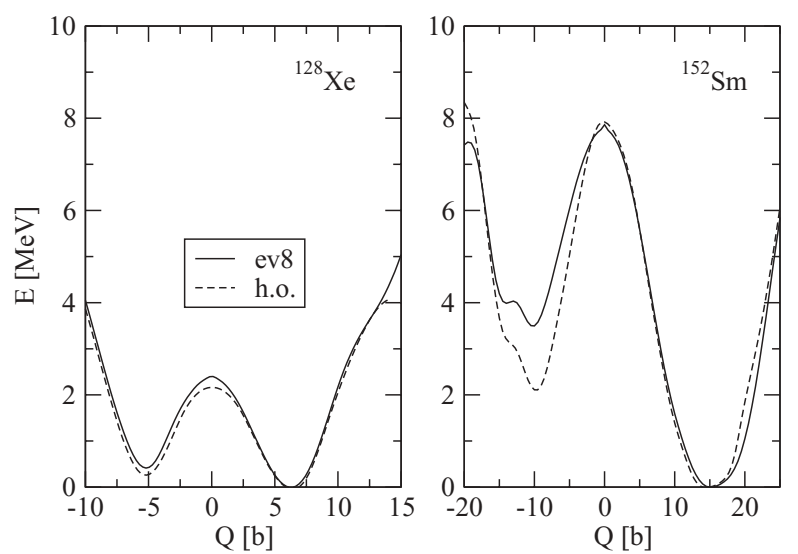

FIG. 1. Potential energy curves obtained with SLy4 and fixed pairing gaps, using different methods for solving the $\mathrm{HF}+\mathrm{BCS}$ equations. 

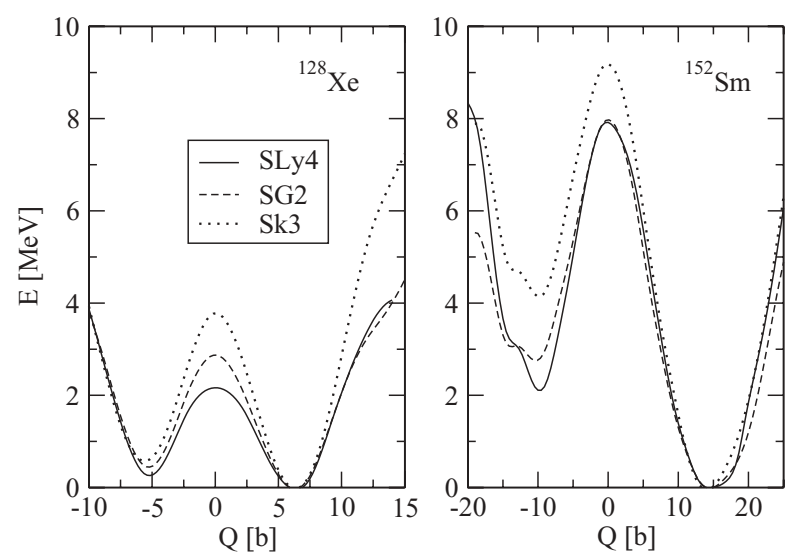

FIG. 2. Potential energy curves obtained with three different Skyrme forces. For details see the main text.

is also apparent if one compares the energy barriers in $\mathrm{Sm}$ isotopes obtained with the parametrization NL3 in Ref. [13], where a constant-gap approach was used, with those in Ref. [15], where a pairing based on the Brink-Boeker part of the Gogny force was used. We should remark that, at variance with Ref. [13], a self-consistent treatment of the pairing is used in Ref. [15]. The main effect is a reduction of the energy barriers in the self-consistent treatment. In the case of nonrelativistic calculations with the Gogny interaction, since the same force is used in both mean-field and pairing channels, a fully self-consistent treatment is possible within a HartreeFock-Bogoliubov formalism. It will be very interesting to explore whether this reduction of the barriers is also present. Work along this line is in progress.

To appreciate the effect on the energy barriers in more detail, we show in Figs. 4, 5, and 6 the results for PECs in ${ }^{96-114} \mathrm{Pd}$, ${ }^{118-136} \mathrm{Xe}$, and ${ }^{120-138} \mathrm{Ba}$ isotopic chains, respectively. The quadrupole deformations corresponding to the ground states of these isotopes can be found in Table I. These chains contain $\mathrm{E}(5)$ candidates found from systematic studies on available data on energy levels $E 2$ and $E 1$, and $M 1$ strengths $[6,8,9,30,31]$.
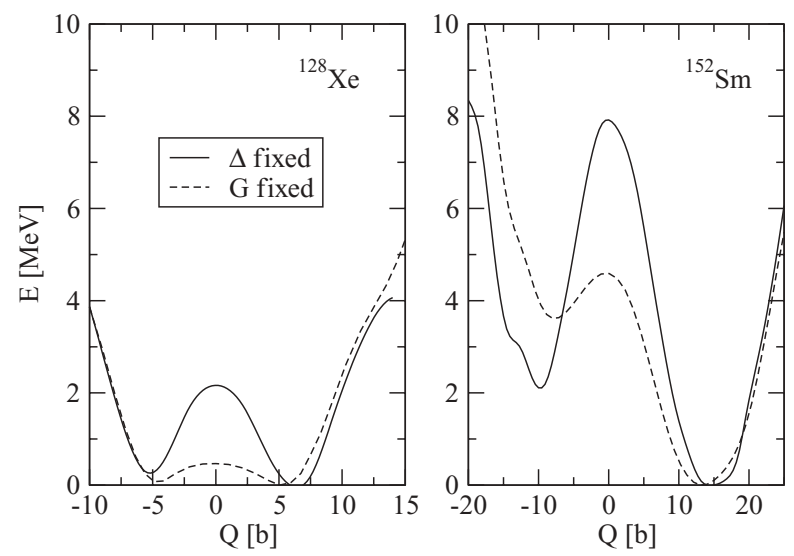

FIG. 3. Potential energy curves obtained with different treatments of pairing. For details see the main text.

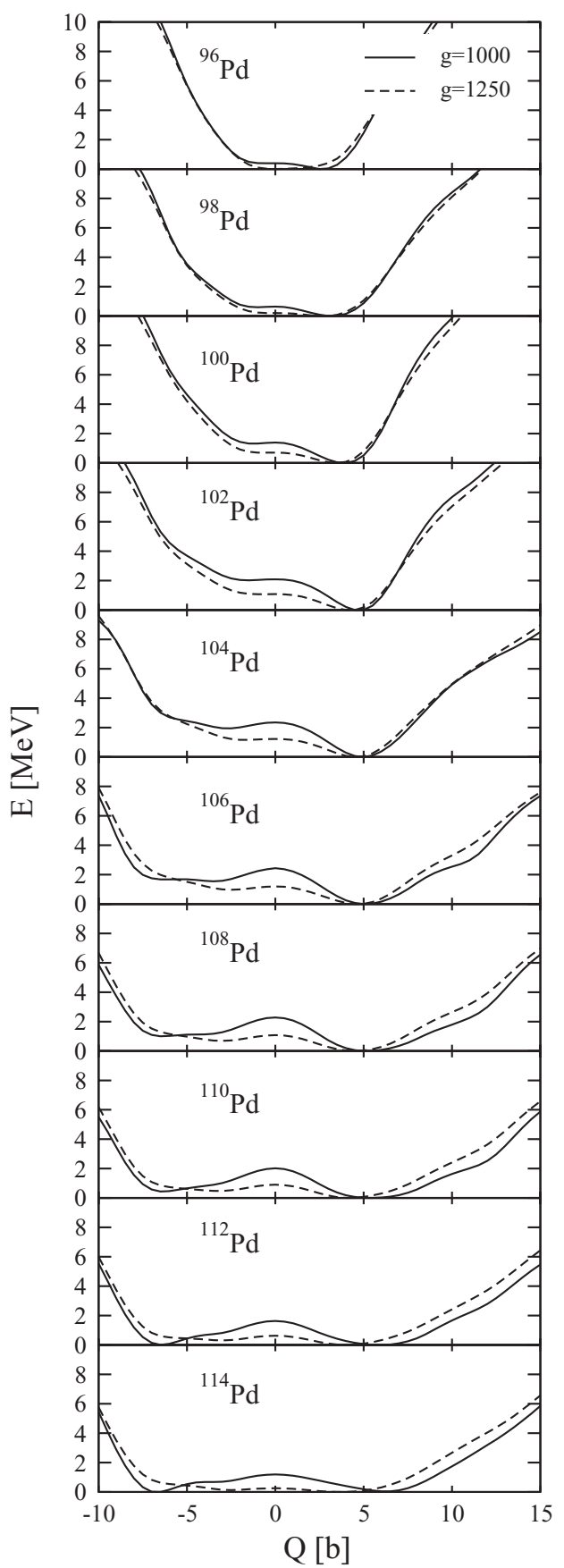

FIG. 4. Potential energy curves in Pd isotopes obtained from constrained $\mathrm{HF}+\mathrm{BCS}$ calculations with the force SLy4 and a zerorange pairing force with two different strengths.

The PECs shown in Figs. 4-6 have been computed within the mean-field scheme based on the Paris-Brussels code EV8, using the parametrization SLy4 and two different choices of the strength $g$ of the zero-range pairing force [Eq. (1)], $g=1000$ and $g=1250 \mathrm{MeV} \mathrm{fm}^{3}$. From these figures, one can see once more that oblate and prolate minima do not change significantly with the strength of pairing in each isotope. The only exceptions worth mentioning are the cases ${ }^{96} \mathrm{Pd},{ }^{114} \mathrm{Pd}$, and ${ }^{134} \mathrm{Xe}$, where we obtain the ground state at different deformations depending on the pairing strength $g$. 


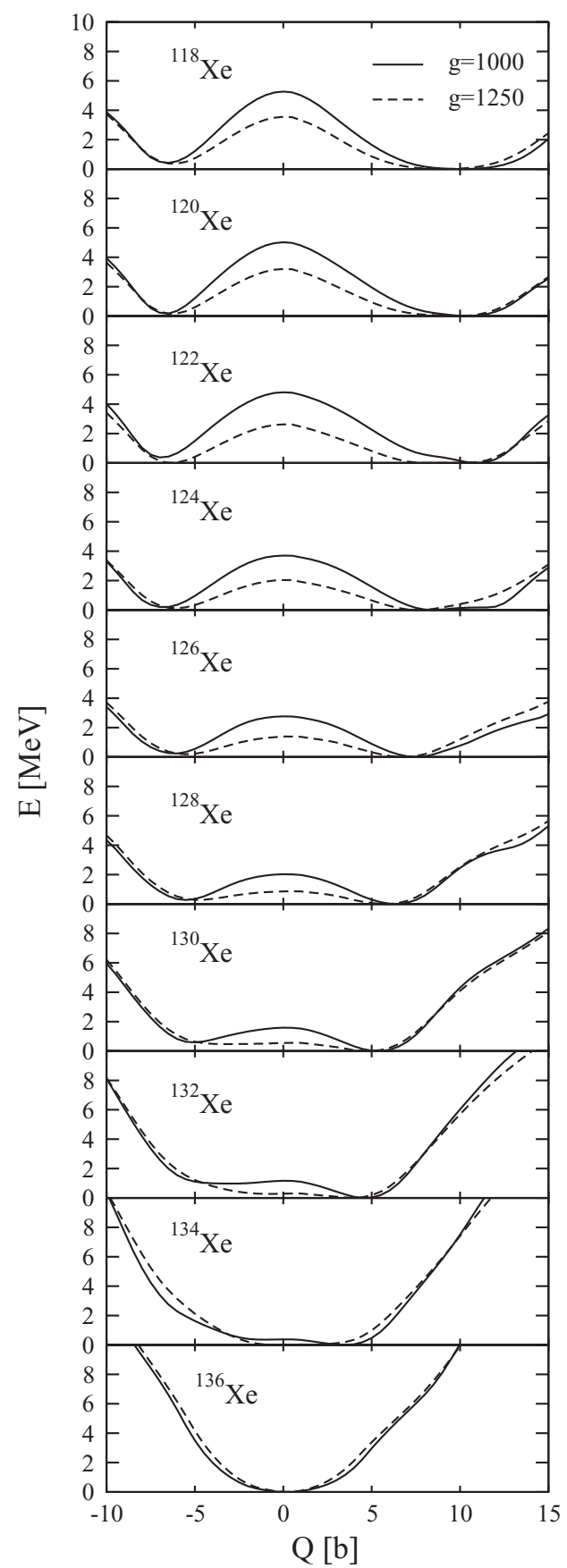

FIG. 5. Same as in Fig. 4, but for Xe isotopes.

This happens because of the flatness of the corresponding PECs, but in any case the energy difference between the minima are always very small. On the other hand, the potential barriers are clearly lower when pairing is stronger. We can also observe the transition from the spherical isotopes ${ }^{96} \mathrm{Pd}(N=$ $50),{ }^{136} \mathrm{Xe}(N=82)$, and ${ }^{138} \mathrm{Ba}(N=82)$ to the $\gamma$-unstable isotopes ${ }^{114} \mathrm{Pd},{ }^{118} \mathrm{Xe}$, and ${ }^{120} \mathrm{Ba}$. We identify the isotopes ${ }^{108,110} \mathrm{Pd},{ }^{128,130} \mathrm{Xe}$, and ${ }^{130,132} \mathrm{Ba}$ as transitional nuclei with rather flat PECs (especially with $g=1250$ ). These results confirm the assumed square well potential in the $\beta$ degree of freedom that leads to the critical point symmetry E(5). They are also in good agreement with the results obtained in Ref. [15]

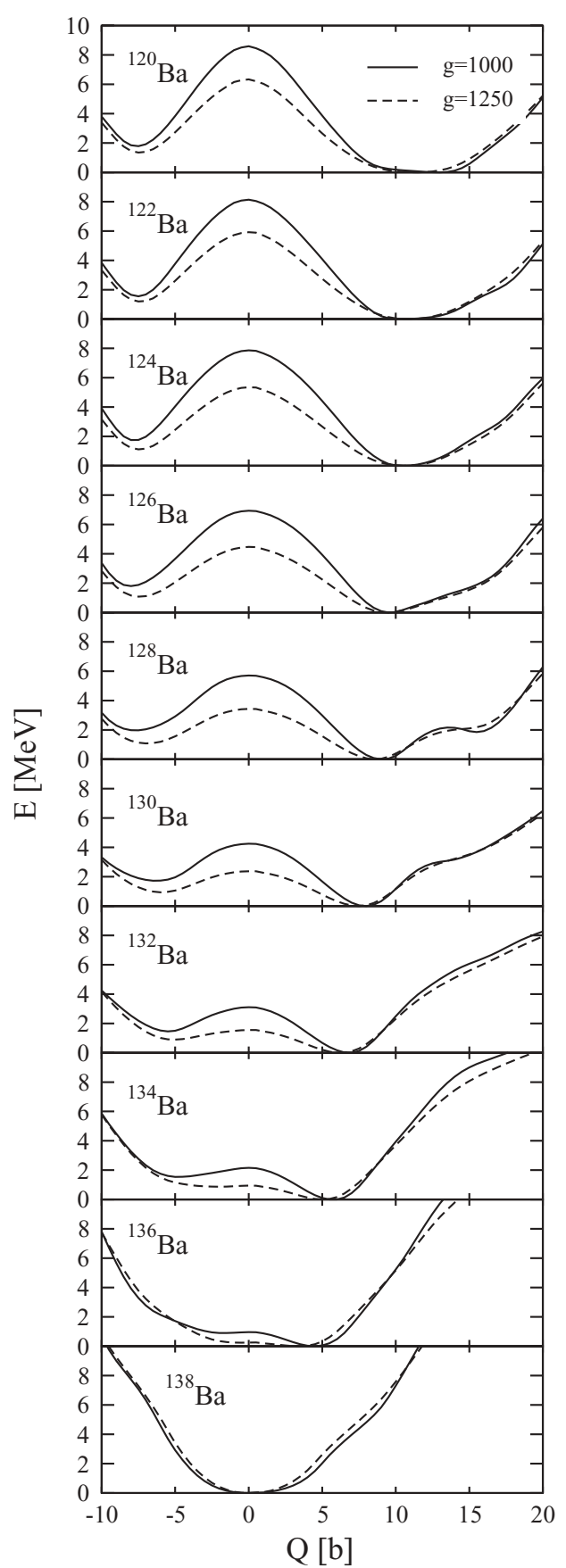

FIG. 6. Same as in Fig. 4, but for Ba isotopes.

using the parametrization NL3 of the relativistic mean-field Lagrangian and a pairing force based on the Brink-Boeker part of the Gogny interaction.

Figure 7 shows the evolution of the energy barriers as a function of the number of neutrons for Xe isotopes for the two values of the pairing strength $g=1000$ and $g=1250 \mathrm{MeV}$ $\mathrm{fm}^{3}$ in [Eq. (1)]. We can observe that the barriers obtained with the strength $g=1250 \mathrm{MeV} \mathrm{fm}^{3}$ are systematically lower than the ones obtained with $g=1000 \mathrm{MeV} \mathrm{fm}^{3}$.

Now we turn the discussion to several rare-earth isotopic chains, $\mathrm{Nd}, \mathrm{Sm}, \mathrm{Gd}$, and Dy, where some of the nuclei $(N=$ 90 isotones) have been identified as exhibiting X(5) behavior, 


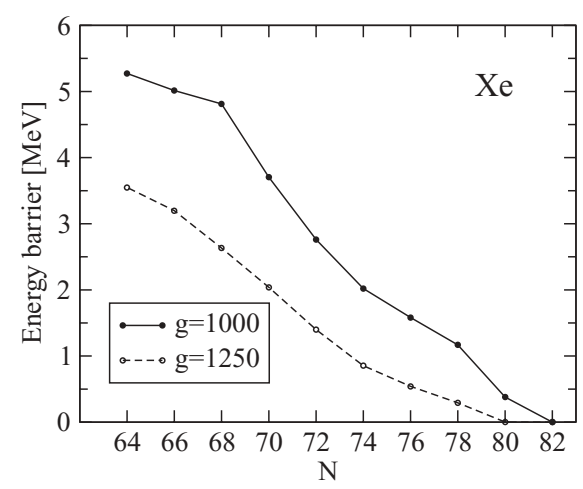

FIG. 7. Energy barriers in Xe isotopes corresponding to the cases shown in Fig. 5.

i.e., a transition between a spherical shape and a well-deformed prolate shape. For example, the nucleus ${ }^{152} \mathrm{Sm}$ [7] was the first identified as exhibiting $\mathrm{X}(5)$ behavior; ${ }^{150} \mathrm{Nd}$ [10] has also been identified. Other candidates have also been suggested with further work (see, for example, Refs. [32-34]).

Figure 8 shows the results for ${ }^{142-156} \mathrm{Nd}$ isotopes $(Z=60)$. The corresponding ground state deformation parameters for these nuclei are given in Table II. We can observe a clear shape transition from spherical ${ }^{142} \mathrm{Nd}(N=82)$ to clearly prolate ${ }^{152-156} \mathrm{Nd}$. The isotopes ${ }^{148} \mathrm{Nd}(N=88)$ and ${ }^{150} \mathrm{Nd}(N=90)$ show a transitional behavior with a rather flat minimum on the prolate side and additional minima on the oblate sector at 3 and $4 \mathrm{MeV}$ excitation energy, respectively. The energy barrier is about $5 \mathrm{MeV}$ in ${ }^{148} \mathrm{Nd}$ and $8 \mathrm{MeV}$ in ${ }^{150} \mathrm{Nd}$.

Figure 9 contains the PECs for ${ }^{144-158} \mathrm{Sm}$ isotopes $(Z=$ $62)$. The corresponding ground state deformation parameters

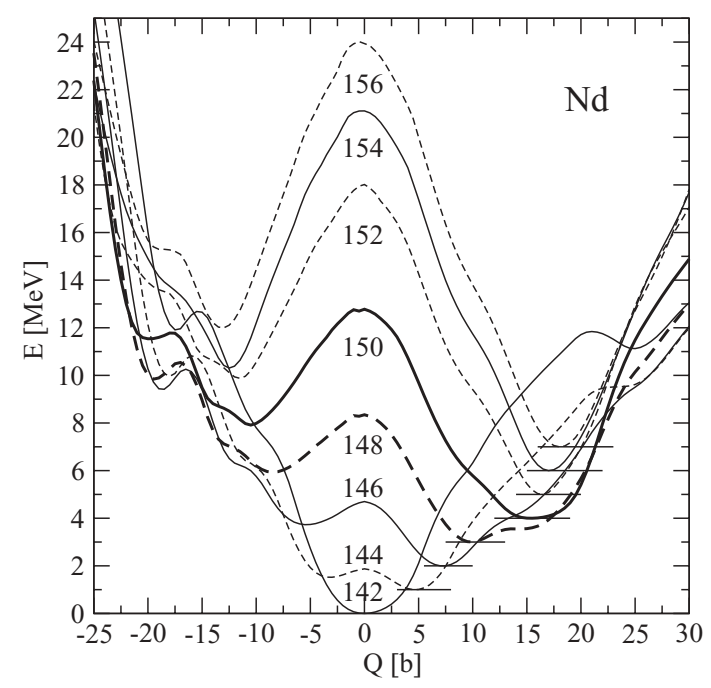

FIG. 8. Constrained HF+BCS calculations in ${ }^{142-156} \mathrm{Nd}$ isotopes with SLy4 and a zero-range pairing force with $g=1000 \mathrm{MeV} \mathrm{fm}^{3}$. For a better comparison, the energies are shifted by $1 \mathrm{MeV}$ for each isotope added, starting from $A=142$. Thick lines correspond to the isotopes that have been suggested to show a critical point symmetry.

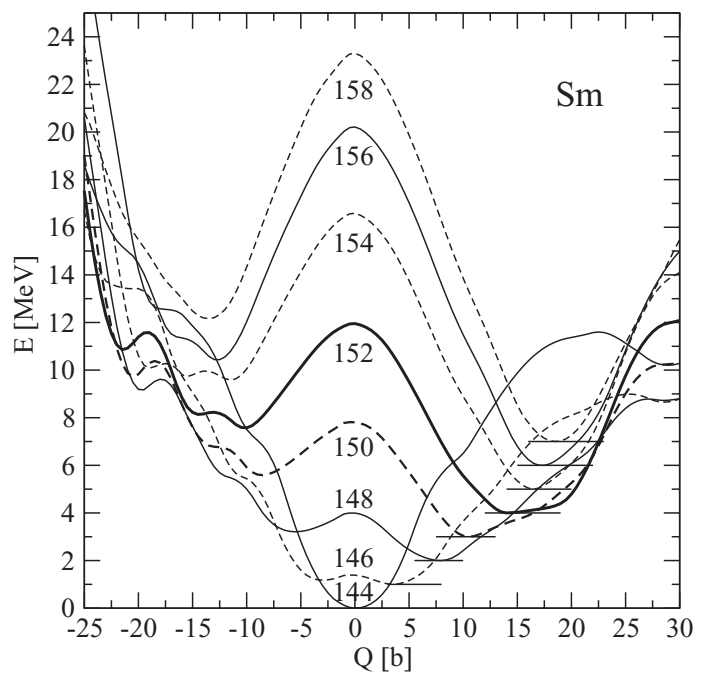

FIG. 9. Same as in Fig. 8, but for Sm isotopes.

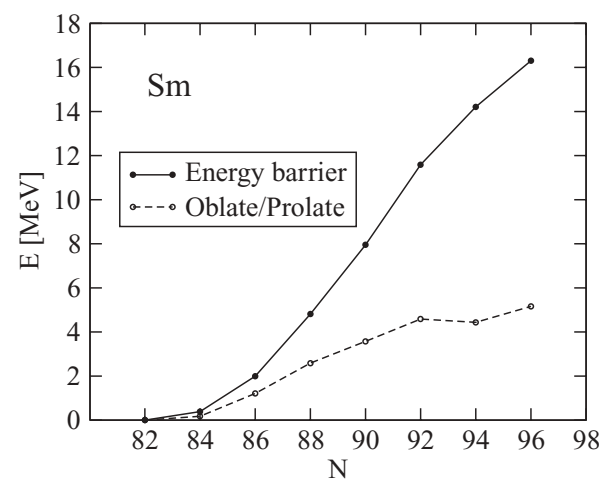

FIG. 10. Energy barriers and energy differences between oblate and prolate minima in the case of Sm isotopes.

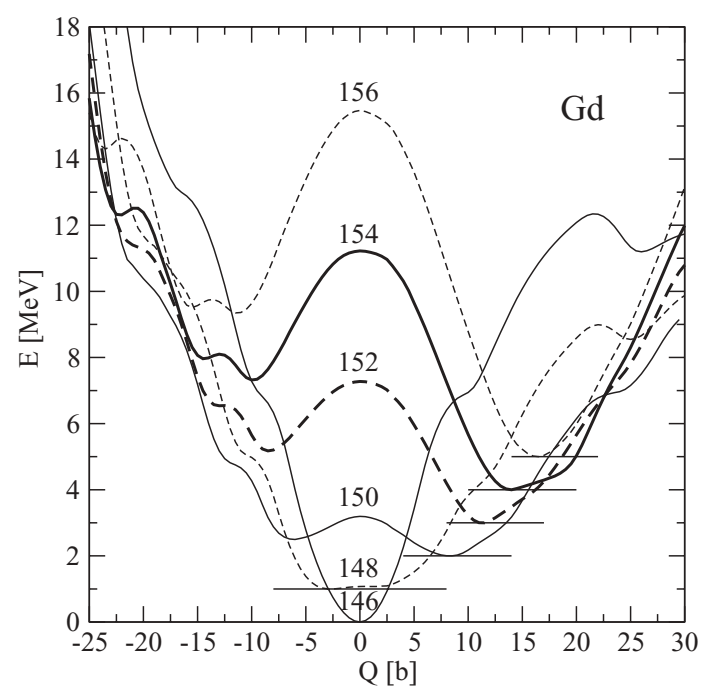

FIG. 11. Same as in Fig. 8, but for Gd isotopes. 


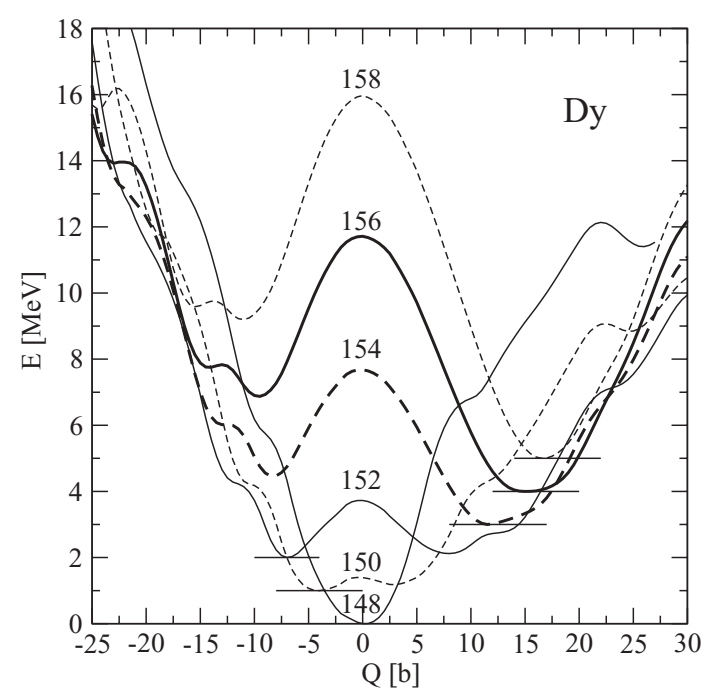

FIG. 12. Same as in Fig. 8, but for Dy isotopes.

are given in Table II. Similar arguments to those in Nd are valid. The transitional behavior between spherical, ${ }^{144} \mathrm{Sm}$, and well prolate deformed, ${ }^{154-158} \mathrm{Sm}$, appears again for the $N=88$ and $N=90$ isotopes, ${ }^{150} \mathrm{Sm}$ and ${ }^{152} \mathrm{Sm}$, respectively. The energy barriers and energy differences between oblate and prolate minima in Sm isotopes are shown in Fig. 10. They are qualitatively similar to the barriers obtained in the other rare-earth isotopic chains. Our PECs for ${ }^{144-158} \mathrm{Sm}$ agree qualitatively with those of Refs. [13] and [15]. They also agree well with the results obtained in Ref. [35] with the Nilsson-Strutinsky + BCS calculations.

The same is true for Gd and Dy isotopes, whose PECs are shown in Figs. 11 and 12, respectively. The ground state deformation parameters for the considered Gd and Dy isotopes are given in Table II. In this case, we find the transitional behavior for $A=152,154$ in $\mathrm{Gd}$ isotopes and $A=154,156$ for Dy isotopes.

From the results described above, we conclude that our calculations do not predict flat PECs for the $N=90$ isotopes, in agreement with previous studies (see, for example, Ref. [15]).

\section{CONCLUSIONS}

In this paper, we have investigated the potential energy curves in a series of isotopic chains containing transitional nuclei that exhibit a critical point symmetry behavior. A microscopic approach based on deformed HF+BCS calculations with Skyrme forces has been used.

We have studied the sensitivity of our results to the effective nucleon-nucleon interaction and to the pairing force. From this analysis, we conclude that the energy barriers in the PECs depend strongly on the details of calculations, especially on the pairing force. This is indeed relevant for this work, because our purpose is to study the extent to which the PECs exhibit a flat behavior at the critical point symmetries.

We have found that the assumptions of flat potentials in the $\mathrm{E}(5)$ critical point symmetry are supported by the present microscopic calculations in ${ }^{108,110} \mathrm{Pd},{ }^{128,130} \mathrm{Xe}$, and ${ }^{130,132} \mathrm{Ba}$, which have been suggested as examples of $\mathrm{E}(5)$. In the case of $\mathrm{X}(5)$, we find that the rare-earth isotopes with $N=88,90$ show a transitional behavior that could be interpreted in terms of X(5) symmetry. However, we do not find a flat behavior, in agreement with previous calculations [13-15].

There remains a long list of tasks to be undertaken in the near future, but the present study could be considered as a first step into a much more systematic exploration of the relation between algebraic models and (nonrelativistic) microscopic models. In particular, it is very important to understand how well the predictions of effective interactions with predictive power all over the nuclear chart compare with those of the already mentioned algebraic models.

\section{ACKNOWLEDGMENTS}

R.R. thanks Prof. F. Iachello (Yale University) for valuable suggestions and discussions. This work was partly supported by Ministerio de Educación y Ciencia (Spain) under Contract No. FIS2005-00640.
[1] J. L. Wood, K. Heyde, W. Nazarewicz, M. Huyse, and P. Van Duppen, Phys. Rep. 215, 101 (1992).

[2] F. Iachello and A. Arima, The Interacting Boson Model (Cambridge University, Cambridge, England, 1987).

[3] F. Iachello, Phys. Rev. Lett. 85, 3580 (2000).

[4] F. Iachello, Phys. Rev. Lett. 87, 052502 (2001).

[5] A. Bohr and B. R. Mottelson, Nuclear Structure (Benjamin, New York, 1975).

[6] R. F. Casten and N. V. Zamfir, Phys. Rev. Lett. 85, 3584 (2000).

[7] R. F. Casten and N. V. Zamfir, Phys. Rev. Lett. 87, 052503 (2001).

[8] N. V. Zamfir et al., Phys. Rev. C 65, 044325 (2002).

[9] R. M. Clark, M. Cromaz, M. A. Deleplanque, M. Descovich, R. M. Diamond, P. Fallon, I. Y. Lee, A. O. Macchiavelli, H. Mahmud, E. Rodriguez-Vieitez, F. S. Stephens, and D. Ward, Phys. Rev. C 69, 064322 (2004).
[10] R. Krucken, B. Albanna, C. Bialik, R. F. Casten, J. R. Cooper, A. Dewald, N. V. Zamfir, C. J. Barton, C. W. Beausang, M. A. Caprio, A. A. Hecht, T. Klug, J. R. Novak, N. Pietralla, and P. von Brentano, Phys. Rev. Lett. 88, 232501 (2002).

[11] D. Tonev, A. Dewald, T. Klug, P. Petkov, J. Jolie, A. Fitzler, O. Moller, S. Heinze, P. von Brentano, and R. F. Casten, Phys. Rev. C 69, 034334 (2004).

[12] A. Dewald et al., Eur. Phys. J. A 20, 173 (2004).

[13] J. Meng, W. Zhang, S. G. Zhou, H. Toki, and L. S. Geng, Eur. Phys. J. A 25, 23 (2005).

[14] Z.-Q. Sheng and J.-Y. Guo, Mod. Phys. Lett. A 20, 2711 (2005).

[15] R. Fossion, D. Bonatsos, and G. A. Lalazissis, Phys. Rev. C 73, 044310 (2006).

[16] P. Bonche, H. Flocard, P. H. -Heenen, S. J. Krieger, and M. S. Weiss, Nucl. Phys. A443, 39 (1985).

[17] M. Bender, P.-H. Heenen, and P.-G. Reinhard, Rev. Mod. Phys. 75, 121 (2003). 
[18] P. Bonche, H. Flocard, and P.-H. Heenen, Comput. Phys. Commun. 171, 49 (2005).

[19] D. Vautherin and D. M. Brink, Phys. Rev. C 5, 626 (1972); D. Vautherin, ibid. 7, 296 (1973).

[20] E. Chabanat, P. Bonche, P. Haensel, J. Meyer, and R. Schaeffer, Nucl. Phys. A635, 231 (1998).

[21] M. Beiner, H. Flocard, N. Van Giai, and P. Quentin, Nucl. Phys. A238, 29 (1975).

[22] N. Van Giai and H. Sagawa, Phys. Lett. B106, 379 (1981).

[23] P. Ring and P. Schuck, The Nuclear Many-Body Problem (Springer, Berlin, 1980).

[24] J. Terasaki, P.-H. Heenen, H. Flocard, and P. Bonche, Nucl. Phys. A600, 371 (1996).

[25] M. Bender, G. F. Bertsch, and P.-H. Heenen, Phys. Rev. C 73, 034322 (2006).

[26] B. Sabbey, M. Bender, G. F. Bertsch, and P.-H. Heenen, Phys. Rev. C 75, 044305 (2007).
[27] H. Flocard, P. Quentin, A. K. Kerman, and D. Vautherin, Nucl. Phys. A203, 433 (1973).

[28] N. Tajima, S. Takahara, and N. Onishi, Nucl. Phys. A603, 23 (1996).

[29] P. Sarriguren, O. Moreno, R. Alvarez-Rodriguez, and E. Moya de Guerra, Phys. Rev. C 72, 054317 (2005).

[30] M. W. Kirson, Phys. Rev. C 70, 049801 (2004).

[31] J. M. Arias, Phys. Rev. C 63, 034308 (2001).

[32] N. V. Zamfir et al., Phys. Rev. C 65, 067305 (2002).

[33] R. M. Clark, M. Cromaz, M. A. Deleplanque, R. M. Diamond, P. Fallon, A. Gorgen, I. Y. Lee, A. O. Macchiavelli, F. S. Stephens, and D. Ward, Phys. Rev. C 67, 041302(R) (2003).

[34] R. F. Casten, N. V. Zamfir, and R. Krucken, Phys. Rev. C 68, 059801 (2003).

[35] J.-Y. Zhang, M. A. Caprio, N. V. Zamfir, and R. F. Casten, Phys. Rev. C 60, 061304(R) (1999). 